\title{
Urologist-operated ultrasound and its use in urological outpatient clinics
}

\author{
This article was published in the following Dove Press journal: \\ Patient Preference and Adherence \\ 21 January 20II \\ Number of times this article has been viewed
}

\section{Mohammad Kazem \\ Moslemi' \\ Behnam Mahfoozi \\ 'Department of Urology, Kamkar Hospital, School of Medicine, Qom University of Medical Sciences, Qom, Iran; ${ }^{2}$ Department of Radiology, Shahid Chamran Hospital, Tehran, Iran}

Correspondence: Mohammad Kazem Moslemi

Department of Urology, Kamkar Hospital, School of Medicine, Qom University of Medical Sciences, Qom, 37I 5694978, Iran Tel +98 9l 22521646

Fax +98251 7713473

Email mkmoslemi@gmail.com
Introduction: Ultrasonograghy plays an important role in the evaluation of urinary tract disorders in cases of medical or surgical renal disorders, because of its lower cost, availability, and lack of ionizing radiation and because with it there is no need for contrast material injection or ingestion. It needs no intervention or preparation and specifically can differentiate between the multiple causes of flank pain. Urologist-operated sonography is a quick, cost-effective, and time-saving modality for both the physician and patient for obtaining first or final diagnosis. Based on its results, patients can be selected for appropriate management and further assessment.

Materials and methods: The efficacy of ultrasound examination by a trained urologist in the differentiation of urological emergencies admitted in a district private clinic was studied. Between April 2008 and April 2010, a total of 724 patients (1448 renal units) had renal ultrasound performed by a trained urologist on acute admission. The sonographic findings were compared with subsequent definitive radiological investigations performed as needed, such as KUB (kidney, ureter, bladder) or IVP (intravenous pyelogram). Patient satisfaction and permission for ultrasonography were evaluated by oral consent. Loin pain was the presenting symptom in $45 \%$ of the patients ( $n=326$ cases).

Results: Diagnosis was achieved in $96 \%$ of patients. Further evaluations were requested as needed in suspicious cases. If any hydronephrosis was detected and patients' history and/or complaints were suggestive of renal or ureteral stones, an outpatient KUB was requested. For more complex situations, IVP was the next option. Abnormal findings were recorded in 184 cases (25.5\%). Mild to moderate unilateral hydronephrosis with or without hydronephrosis was the most common finding observed sonographically. The sensitivity of our ultrasonography evaluation was $99.7 \%$.

Conclusion: Office urologist-operated sonograghy may supplement the information available through routine history, physical examination, and laboratory studies. Our study shows that urological trainees can use ultrasound with high levels of accuracy, thereby improving patient management with a high level of patient satisfaction.

Keywords: ultrasonograghy, kidney, bladder, urologist, CT scan, cystourethroscopy, KUB (kidney, ureter, bladder), urologist-operated sonography (UOS)

\section{Introduction}

The value of urologist-operated ultrasound scanning was assessed in a urological outpatient clinic. In terms of accuracy, the urologists' ultrasound scans of the genitourinary tract proved comparable with those of radiologists. The techniques were quickly learned, and scanning added little to the consultation time. Scanning during clinic time was shown to be both time saving and cost effective by allowing more rapid assessment of the patient and a speedier diagnosis. ${ }^{1}$ In economic terms, considerable savings could be made by reducing the need for formal ultrasound referrals, as patients scanned at the outpatient clinic would be spared two further trips to hospital, one for the formal examination and 
another for the outpatient clinic review. Clinic turnover thus would become more efficient, and inconvenience to the patient and transport costs would be lessened. ${ }^{1}$

Since its first clinical applications in the 1940s, steady advances in ultrasound technology have continued to expand its role in the diagnosis, management, and follow-up of patients with urologic disorders. ${ }^{2,3}$ Ultrasound is based on the interpretation of sound waves that have been reflected by the interface of different tissues in the body. ${ }^{3}$ The role of ultrasound in urological investigation is well established. ${ }^{1,4}$ Two-dimensional ultrasound is the technique most familiar to practising urologists. In this technique the reflected echoes appear as bright spots on the readout, with signal intensity being proportional to the brightness.

The urologist is the professional most capable of obtaining the maximum effectiveness of this technique so that the availability of ultrasound machines in urology departments rationalizes and limits the demand of ultrasound tests and diminishes significantly the indication of radiological tests and the number of cystoscopies. The urinary tract is readily imaged with ultrasound, and increasing numbers of urologists are using upper tract imaging to localize renal stones prior to extracorporeal shockwave lithotripsy. ${ }^{6}$

This study was specifically designed to evaluate the ability of urologists to detect urinary tract abnormalities in routine urological referrals.

\section{Materials and methods}

Scans were performed by a urologist who had attended a 3-day focused ultrasound examination instruction course. All scans were videotaped and subsequently reviewed by a radiologist. Between April 2008 and April 2010, a total of 724 patients (421 males: 58\%, 303 females: $42 \%$; see Table 1), mean age 57 years (range 1-87 years), attending a district outpatient private urology clinic underwent an ultrasound examination of the urinary tract using an Emperor 3.5 MHz portable ultrasound machine in the clinic. All kidney units were assessed for collecting system dilatation, cortical thickness, and the presence of intrarenal masses. The patients' indications for ultrasonograghy were flank pain, evaluation of kidney hydronephrosis, evaluation of impact of lower urinary tract symptoms (LUTS) on the kidneys or bladder, and detection of space-occupying lesions (SOLs). Of the cases, 642 (88.5\%) were adults and $82(11.5 \%)$ pediatric.

Each eligible patient was examined after a detailed medical history had been taken. The average time spent on every sonographic evaluation was 5 minutes. For more definite
Table I Total number of patients and demographic data

\begin{tabular}{lllll}
\hline $\begin{array}{l}\text { Total } \\
\text { number }\end{array}$ & Male (\%) & Female (\%) & Adults (\%) & Pediatric (\%) \\
\hline 724 cases & 421 & 303 & 642 & 82 \\
& cases (58) & cases (42) & cases (88.5) & cases (11.5) \\
\hline
\end{tabular}

diagnosis, 85 KUBs (kidney, ureter, bladder), 26 IVPs (intravenous pyelograms), 12 computed tomography (CT) scans, and three cystoscopies were performed as follow-up imaging. Indications for ultrasonography (Table 2) in our eligible cases were flank pain $45 \%$, evaluation of benign prostatic hyperplasia (BPH) and/or LUTS symptoms $26 \%$, urolithiasis $12 \%$, evaluation of SOLs of the kidney $7 \%$, evaluation of bladder tumors $7 \%$, and evaluation of congenital anomalies $3 \%$. The patients' satisfaction in terms of time to final diagnosis, convenience, and effective and proper treatment or referral was assessed by oral consent. All the patients were suggested to select urologist- or radiologist-operated sonography (UOS). The results were analyzed by SPSS software, Version 16 (SPSS Inc., Chicago, IL, USA).

\section{Results}

Complete or partial diagnosis by ultrasound was achieved in $96 \%$ (695 patients). We carried out further investigations for more precise evaluations in cases of incomplete diagnosis and also in the remaining 29 patients (4\%) with no diagnosis. If any hydronephrosis was detected or patient history or complaint suggestive of renal or ureteral stones, an outpatient KUB was requested. For more complex situations, such as ovarian tumors or cervical or colon cancer with secondary obstructive uropathies, IVP and/or abdominopelvic CT scan were the next options.

In 184 patients $(25.5 \%)$, some urinary system abnormality was found in which ultrasound findings were confirmed by further investigations with KUB, IVP, and CT scan in 123 cases (Table 3 ). The six discordant results were four false positives and two false negatives (small renal stones were found in the false-negative cases). Sensitivity was $99.7 \%$ and

Table 2 Indications for ultrasonography in our eligible cases

\begin{tabular}{lll}
\hline Indication & Number of cases & Percentage \\
\hline Flank pain & 326 & 45 \\
BPH and/or LUTS symptoms & 188 & 26 \\
Urolithiasis & 87 & 12 \\
SOLs of the kidney & 50 & 7 \\
Evaluation of bladder tumors & 50 & 7 \\
Evaluation for congenital & 23 & 3 \\
anomalies & &
\end{tabular}

Abbreviations: BPH, benign prostatic hyperplasia; LUTS, lower urinary tract symptoms; SOLs, space-occupying lesions. 
positive predictive value was $99.5 \%$. Two patients with a small bladder tumor seen with ultrasonography were shown to have a normal bladder with cystourethroscopy, and in two other cases no hydronephrosis was found. Abnormal findings were recorded in 184 cases $(25.5 \%)$. Mild to moderate unilateral hydronephrosis was found in 93 cases $(50.5 \%)$, unilateral or bilateral renal stones in 47 cases (25.5\%), severe unilateral hydronephrosis in 21 cases (11.4\%), and bilateral hydronephrosis in five cases $(2.7 \%)$ (bladder stones $4.8 \%$, bladder tumor $3.8 \%$, and renal tumor $1 \%$; Table 4 ).

All of the patients were satisfied with UOS in terms of cost and time saving and rapid diagnosis and treatment (100\%). All patients selected UOS voluntarily except for one patient for religious reasons.

\section{Discussion}

Bedside ultrasonography is a rapid, safe, and noninvasive imaging system for the evaluation of urinary obstruction and has the ability not only to detect urinary obstructions but also to exclude other abdominal pathologies such as abdominal aneurysms, free fluid, and gallstones. ${ }^{7-9}$ Diagnostic accuracy in UOS means ruling out/in some causes of flank pain by diagnosing the presence or absence of hydronephrosis or bladder stones in the case of BPH for whether or not to proceed with BPH surgery. Diagnostic accuracy is achieved by following the patient or from further evaluations such as KUB, IVP, or CT scans.

Patients presenting as emergencies with loin pain can cause considerable diagnostic difficulty, as clinical history and examination are unreliable in the diagnosis of renal or ureteric colic. The most common cause of acute flank pain in adults is passage of a renal stone or acute pyelonephritis. ${ }^{10}$ Although it is not possible to identify calculi in the ureter ultrasonographically, mild to moderate dilatation of the pelvicalyceal system on the side of the pain can be taken as evidence of an obstructed ureter due to calculus ${ }^{11}$ unless proved otherwise. Ultrasound, which is widely available in urology, ${ }^{2,12}$ could prove useful as an admission screening test in order to detect upper urinary tract pathology, identifying patients

Table 3 Overall investigations performed in our cases besides primary ultrasonography for further evaluation

\begin{tabular}{ll}
\hline Type of procedure & Total number of cases \\
\hline Ultrasonography & 724 \\
KUB & 85 \\
IVP & 26 \\
CT scan & 12 \\
Cystourethroscopy & 3 \\
\hline
\end{tabular}

Abbreviations: $\mathrm{CT}$, computed tomography; IVP, intravenous pyelogram; $\mathrm{KUB}$, kidney, ureter, bladder.
Table 4 Abnormal findings detected

\begin{tabular}{ll}
\hline Abnormal findings & $\begin{array}{l}\text { Number of cases } \\
\text { (total I84) (\%) }\end{array}$ \\
\hline Mild to moderate unilateral hydronephrosis & $93(50.5)$ \\
Severe unilateral hydronephrosis & $21(11.4)$ \\
Bilateral hydronephrosis & $5(2.7)$ \\
Unilateral or bilateral renal stones & $47(25.5)$ \\
Bladder stones & $9(4.8)$ \\
Bladder tumor & $7(3.8)$ \\
Renal tumor & $2(1)$ \\
\hline
\end{tabular}

requiring intravenous urography. Despite the introduction of low osmolarity contrast agents, there is still a small but significant risk of contrast reaction, including life-threatening anaphylaxis. Prior selection by ultrasonograghy will reduce the number of patients unnecessarily exposed to this risk.

Ultrasound provides a safe and reliable working diagnosis for immediate management in renal colic and obviates the necessity for out-of-hours emergency intravenous studies with their inherent problems. Ultrasound is known to be highly operator dependent, ${ }^{4}$ so the definition of dilatation can be difficult. It is important to remember that renal unit dilatation is not an invariable consequence of ureteric obstruction; nevertheless, obstruction should always be considered even in its absence.

Ultrasound has proved to be very accurate in evaluating patients with common urologic problems such as renal obstruction and urinary infection and in screening for uropathology among children with siblings known to have urologic disease. The benefits of ultrasound in adult and pediatric populations include diagnostic accuracy, ease of use, absence of radiation exposure, and no risk of adverse reactions to contrast agents. Office-based urologist-operated ultrasound supplements the information elicited from routine history, physical examination, laboratory studies, and other radiologic investigations. ${ }^{13}$ In a study of 50 patients by Nargund et al, it was shown that sonography by urologists has $97 \%$ specificity and $84 \%$ sensitivity. ${ }^{14}$

The left kidney is more difficult to visualize, this occurs because of overlying bowel gas or air in the stomach that reflects sound waves, the more superior location of the left kidney, and the absence of the liver to provide an acoustic window. In fact, the test characteristics were better when the right kidney was examined. ${ }^{15}$ However, in our study, these differences were not technically significant.

When evaluating renal masses, differentiating cysts from solid lesions is the primary role of ultrasound. Ultrasonography is also helpful and frequently superior to $\mathrm{CT}$ in demonstrating the complex internal architecture of cystic lesions in terms of internal fluid content, septations, 
tiny nodules, and wall abnormalities, including associated soft tissue masses. ${ }^{9}$ The primary role of ultrasonography in evaluating benign cystic renal disease is the distinction of a simple cyst from a solid mass and in defining the characteristics of a complex cyst. ${ }^{16}$

The endpoint in UOS is determination of renal cortical thickness, renal dimensions, presence or absence of hydronephrosis and its severity (mild, moderate, or severe), determination of renal stones by finding their acoustic brightness and postacoustic shadows and their differentiation by other bright echoes, and the presence or absence of renal SOLs.

In the case of the bladder, determination of bladder wall thickness, bladder stones, and prostate volume measurement and heterogeneity, determination of bladder SOL-like tumors is important.

Ultrasonograghy should be performed by the clinician only after appropriate training. So, after at least 2-6 weeks, every urologist can essentially be capable of doing UOS. Office-based UOS should not replace the proper evaluation of patients by a radiologist who is trained specifically to make diagnoses using this modality. However, for quick, efficient evaluation of the patient to uncover a disease process, office-based UOS may supplement the information available through routine history, physical examination, and laboratory studies. ${ }^{17}$

\section{Conclusion}

Abdominopelvic sonography performed in urological outpatient clinics on unprepared patients was the only investigation necessary for the evaluation of common problems such as nonspecific urinary symptoms, flank pain, recurrent urinary tract infections, and several causes of lower urinary tract symptoms. The results of this study provide convincing evidence that access to ultrasound should be made freely available in emergency rooms and outpatient urology clinics. Based on our study results, we found that UOS should be used routinely without any limitation in every private/primary/secondary urological center.

\section{Disclosure}

The authors report no conflicts of interest in this work.

\section{References}

1. Vesey SG, Lumb GN, O’Boyle PJ. An evaluation of urologist-operated ultrasound and its use in the urological out-patient clinic. Br J Urol. 1988;61(1):74-76.

2. Resnick MI. Ultrasonography of the prostate and testes. J Ultrasound Med. 2003;22:869-877.

3. Singer EA, Golijanin DJ, Davis RS, Dogra VD. What's new in urologic ultrasound? Urol Clin North Am. 2006;33(3):1-10.

4. Lewis-Jones HG, Lamb GHR, Hughes PL. Can ultrasound replace the intravenous urogram in preliminary investigation of renal tract disease? A prospective study. Br J Radiol. 1989;62:977.

5. Resnick MI, Novick AC. Ultrasonography. M.I. A.C. Urology Secrets. Philadelphia: Hanley and Belfus; 1999:14.

6. Kiely EA, Madigan D, Ryan PC, Butler MR. Ultrasonic imaging for extracorporeal shockwave lithotripsy: analysis of factors in successful treatment. Br J Urol. 1990;66:127.

7. Noble VE, Brown DFM. Renal ultrasound. Emerg Med Clin N Am. 2004;22:641-659.

8. Marston WA, Ahlquist R, Johnson G Jr, Meyer AA. Misdiagnosis of ruptured abdominal aortic aneurysms. J Vas Surg. 1992;16(10): $17-22$.

9. Eskelinen M, Ikonen J, Lipponen P. Usefulness of history-taking, physical examination and diagnostic scoring in acute renal colic. Eur Urol. 1998;34(6):467-473.

10. Bishop NL. The influence of emergency urography and hematuria on the diagnosis of ureteric colic. Clin Radiol. 1980;31:605.

11. Hill MC, Rich JI, Mardiat JG, Finder CA. Sonography versus excretory urography in acute flank pain. Am J Radiol. 1985;144:1235.

12. Reisman EM, Kennedy TJ, Roehrborn CG, McConnell JD. A prospective study of urologist-performed sonographic evaluation of the urinary tract in patients with prostatism. $J$ Urol. 1991;145:1189.

13. Donovan JM, Ney KG, Maizels M. Urosound. In-office ultrasonography for pediatric urology. Urol Clin North Am. 1989;16(4):841-855.

14. Nargund VH, Cumming JA, Jerwood D, et al. Ultrasound in urological emergency: results of self audit and implications for training. Int Urol Nephrol. 1996;28(3):267-271.

15. Rosen CL, Brown DF, Sagarin MJ, Chang Y, McCabe CJ, Wolfe RE. Ultrasonography by emergency physicians in patients with suspected ureteral colic. J Emerg Med.1998;16(6):865-70.

16. Therese MW. Sonography of benign renal cystic disease. Ultrasound Clin. 2006;1:15-24.

17. Acino S, Resnick MI. Office urologic ultrasound. Urol Clin North Am. 1988;15(4):577-588.
Patient Preference and Adherence

\section{Publish your work in this journal}

Patient Preference and Adherence is an international, peer-reviewed, open access journal focusing on the growing importance of patient preference and adherence throughout the therapeutic continuum. Patient satisfaction, acceptability, quality of life, compliance, persistence and their role in developing new therapeutic modalities and compounds to

\section{Dovepress}

optimize clinical outcomes for existing disease states are major areas of interest. This journal has been accepted for indexing on PubMed Central. The manuscript management system is completely online and includes a very quick and fair peer-review system. Visit http://www.dovepress.com/ testimonials.php to read real quotes from published authors. 